\title{
H.-J. Biersack, L. M. Freeman (eds): Clinical nuclear medicine
}

\author{
Springer, New York, 2007, 548 pp, 310 illustrations, 263 in colour, hardcover, \\ ISBN: 978-3-540-28025-5; Euro 213.95
}

\author{
Ignasi Carrió
}

Published online: 20 August 2009

(C) Springer-Verlag 2009

This is a comprehensive textbook that covers all relevant clinical applications in nuclear medicine. This book represents a substantial effort to recognize the dramatic advances in clinical nuclear medicine and the new directions in diagnosis and therapy in which the field is heading. The editors and authors, from both sides of the Atlantic, bring a very balanced view of clinical nuclear medicine at a global scale. The book benefits from contributions from international leaders in the field that fill the chapters with vivid illustrations in colour. Such carefully selected experts review the field from its cornerstones to the advances that will occupy clinical nuclear medicine in the next years.

Readers will note that this new multiauthored text has tried to move away from orthodox texts. The authors have attempted to provide a comprehensive but succinct anatomical, pathophysiological and molecular basis for the primary and secondary disorders addressed throughout the book, and use the pathogenetic basis to explain the evolution of the new diagnostic and therapeutic strategies employed in nuclear medicine. "Clinical Nuclear Medicine" is not just another thick textbook; it is easy to handle and consult. I am sure the readers will highly appreciate the achievement of the editors in putting together a book of such manageable size and conciseness.

The book presents in careful order the most up-to-date and comprehensive clinical applications in nuclear medicine. It provides the practitioner with the current clinical state-of-the-art and indicates the newest directions in the field, including both the technological and biological advances. To achieve such goals in a manageable book, the contents are divided into 30 chapters grouped into three specific sections. Section 1 addresses in detail physics, instrumentation, radiation protection issues, followed by the essentials in radiochemistry and radiopharmacy. Section 2 focuses on nuclear medicine diagnosis covering each organ system and disease entity. Section 3 focuses on nuclear medicine therapy including all current clinical applications and most recent developments. Interestingly, the different nuclear medicine technologies, including PET, are incorporated into each organ system or disease-specific section rather than being presented in a separate chapter as has been done classic textbooks.

The unparalleled advances in nuclear medicine over the years have been supported by rigorous basic and clinical research. Throughout the book, the authors avoid excessive technicalities and stick to the knowledge that is relevant for the clinical applications in the field. Figures and diagrams are clear and informative. The authors have purposely avoided inclusion of details and experimental data or results of trials which are available to the readers at the click of the mouse.

I believe that this is a very good textbook for nuclear medicine practitioners, but in particular it will attract nuclear medicine residents and fellows and entice them to the field.

I. Carrió $(\bowtie)$

Department of Nuclear Medicine, Hospital Sant Pau,

Barcelona, Spain

e-mail: icarrio@santpau.cat 